\title{
Micellar Catalysed Kinetic Oxidation Study of Acetophenone by $N$-Bromosaccharin in Aqueous Acetic Acid Medium
}

\author{
V. K. SHARMA, ILA JAIN ${ }^{*}$, PREETI SINGH and K. SHARMA \\ Department of Chemistry, Govt. Motilal Vigyan Mahavidyalaya, Bhopal, M.P. India \\ ilajain69@gmail.com
}

Received 12 June 2015 / Accepted 26 June 2015

\begin{abstract}
The kinetic oxidation study of acetophenone by $N$-bromosaccharin in aqueous acetic acid medium and in presence of $\mathrm{Hg}(\mathrm{II})$ acetate was investigated in the presence of both cationic and anionic micelles. The reaction obeys first order kinetics in oxidant both in the presence as well as absence of micelle. The kinetic results for dependence on substrate concentration indicate intermediate complex formation between oxidant and substrate species. The reactions are acid catalysed and follow both acid dependent and independent path. Change in polarity of the medium, effect of addition of saccharin and $\mathrm{Hg}$ (II) acetate have also been investigated. Both type of micelles catalyses the oxidation. Binding parameters have been calculated by analyzing the data using the model suggested by Piszkiewicz. On the basis of kinetic results probable mechanism is proposed.
\end{abstract}

Keywords: Kinetics, Oxidation, Acetophenone, $N$-Bromosaccharin, Micelles

\section{Introduction}

Micelle catalysed reactions have been the area of current research. The rate effect caused by the micelles is a composite of non-covalent interactions between the micelles on one hand and the reactant and activated complex on the other hand. Oxidation kinetics of acetophenone and substituted acetophenones by different oxidants viz. potassium

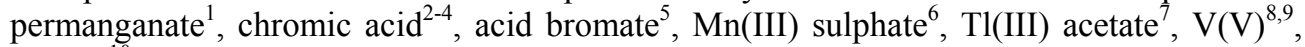
$\mathrm{Ce}(\mathrm{IV})^{10}$ have been reported. The present investigation is carried out to study the catalytic effect of both cationic and anionic surfactants on the $N$-bromosaccharin oxidation of acetophenone in aqueous acetic acid medium in the presence of $\mathrm{Hg}$ (II) acetate as scavenger for $\mathrm{Br}^{-}$.

\section{Experimental}

$N$-Bromosaccharin was prepared by the method suggested by Bacchawat and Mathur ${ }^{11}$ and was standardized by iodometric method ${ }^{12}$. Acetophenone (Rhone-Poulene, b.p. $202{ }^{\circ} \mathrm{C}$ ) solution was prepared by dissolving in glacial acetic acid. The solution of cetyltrimethylammonium bromide (CTAB)- cationic surfactant and sodium lauryl sulphate 
(NaLS)- anionic surfactant was prepared by dissolving their appropriate amount in distilled water. Acetic acid (Sd.fine SDS) was first refluxed with chromium trioxide (20 g) and acetic anhydride $(20 \mathrm{~mL})$ for $3 \mathrm{~h}$ using air condenser. Then it is distilled above $110{ }^{\circ} \mathrm{C}$ and distillate was collected. This distillate was used in kinetic studies.

\section{Kinetic measurements}

Kinetic studies were carried out using Ostwald's isolation method. The reaction mixture was equilibrated in a thermosat $\left( \pm 0.1{ }^{\circ} \mathrm{C}\right)$ at desired temperature. The progress of the reaction was monitored by iodometric estimation of unreacted $N$-bromosaccharin.

\section{Stoichiometry and product analysis}

Stoichiometry of the reaction was ascertained by equilibrating the reaction mixture containing an excess of $N$-bromosaccharin $(\approx 10$ times) over the concentration of acetophenone and the concentration of acetic acid and mercuric acetate was maintained at experimental runs. Stoichiometric studies revealed that $1 \mathrm{~mol}$ of acetophenone consumed 2 mols of NBSA in the presence as well as absence of surfactant. The stoichiometric equation can be written as:

$\mathrm{C}_{6} \mathrm{H}_{5} \mathrm{COCH}_{3}+2 \mathrm{C}_{6} \mathrm{H}_{4} \mathrm{COSO}_{2} \mathrm{NBr}+\mathrm{H}_{2} \mathrm{O} \longrightarrow \mathrm{C}_{6} \mathrm{H}_{5} \mathrm{COCHO}+2 \mathrm{C}_{6} \mathrm{H}_{4} \mathrm{COSO}_{2} \mathrm{NH}+2 \mathrm{HBr}$

The product phenyl glyoxal was identified by the formation of yellow phenyl hydrazide.

\section{Results and Discussion}

The kinetics of acetophenone oxidation by $N$-bromosaccharin in the presence and absence of $\mathrm{CTAB}$ and NaLS was carried out under the condition [Acetophenone] and [Hg(II)] $>>$ [NBSA].

\section{Dependence of rate on oxidant concentration}

The reaction follows first order kinetics in oxidant (Table 1) as revealed by the linear plots between $\log (\mathrm{a}-\mathrm{x})$ and time under both in the presence as well as absence of micelle. However, on the variation of initial concentration of $N$-bromosaccharin the pseudo first order rate constant decreases with the increase in the concentration of oxidant. Such a decrease in rate constant value suggests that $N$-bromosaccharin is involved in a preequilibrium with some nucleophilic species (probably $\mathrm{H}_{2} \mathrm{O}$ ) to form some intermediate with lower reactivity. Similar results were reported in the oxidation of benzhydrols ${ }^{13}$, primary aliphatic alcohols ${ }^{14}$, benzyl alcohol ${ }^{15}$ and $\alpha$ - hydroxyl acids ${ }^{16-18}$.

\section{Dependence of rate on substrate concentration}

The pseudo first order rate constant increases with increase in the concentration of ketone (Table 1). However, the value of second order rate constant, $\mathrm{k}_{2}\left(\mathrm{k}_{2}=\mathrm{k}_{1} /\right.$ [ketone]) does not show constancy. Both in the presence and absence of surfactant the plot between $\mathrm{k}_{1}$ and [ketone] is initially (at lower concentration) linear passing through origin and then bends towards $\mathrm{x}$-axis at higher concentration of ketone (Figure 1). It suggests that the order with respect to substrate in both the systems varies from 1 to 0 . For each system the double reciprocal plot between $\mathrm{k}_{1}$ and [ketone] is linear with positive intercept on $\mathrm{y}$-axis giving a kinetic evidence for complex formation.

\section{Dependence of rate on the concentration of micelles}

The nature of the plot between $\mathrm{k}_{1}$ and surfactant concentration (Figure 2) suggests that at lower concentration of surfactant, reaction rate increases with increase in the concentration of each surfactant, CTAB and NaLS and then at higher concentration after cmc the rate decreases attaining a limiting value in NaLS while it is almost constant in CTAB. The rate[surfactant] profile suggests that each oxidation, under study, is micellar catalysed (Table 1). 


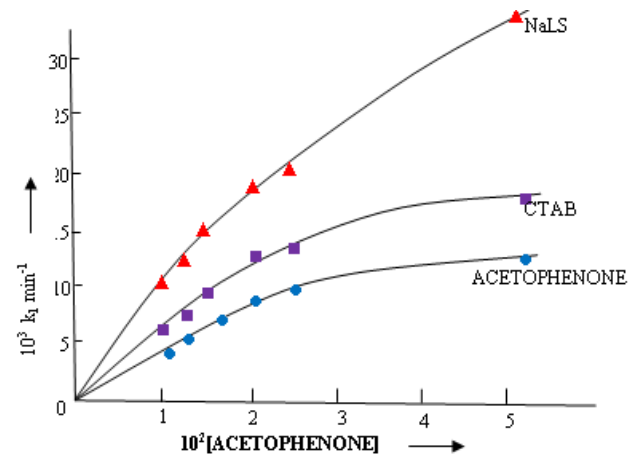

Figure 1. Dependence of rate on [Acetophenone]

$[N B S A]=2.0 \times 10^{-3} \mathrm{M},\left[\mathrm{Hg}(\mathrm{OAc})_{2}\right]=1.0 \times 10^{-2} \mathrm{M}, \mathrm{HOAc}-\mathrm{H}_{2} \mathrm{O}=20 \%(\mathrm{v} / \mathrm{v}),[\mathrm{CTAB}]=5.0 \times 10^{-6} \mathrm{M}$, $[\mathrm{NaLS}]=6.0 \times 10^{-6} \mathrm{M}, \mathrm{Temp}=50^{\circ} \mathrm{C}$

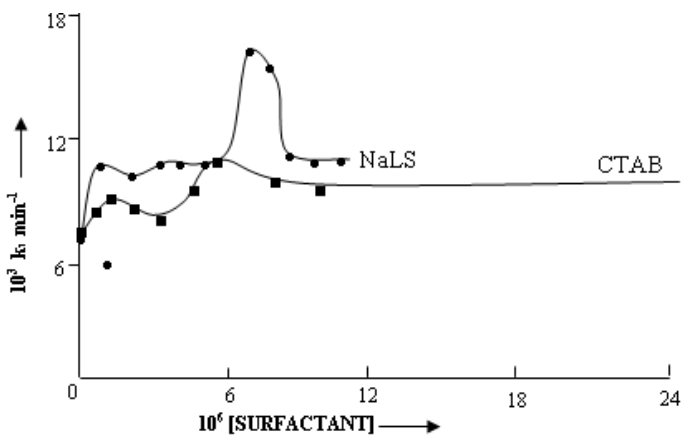

Figure 2. Dependence of rate on [Surfactant]

$[$ NBSA $]=2.0 \times 10^{-3} \mathrm{M},[$ Acetophenone $]=2.0 \times 10^{-3} \mathrm{M},\left[\mathrm{Hg}(\mathrm{OAc})_{2}\right]=1.0 \times 10^{-2} \mathrm{M}, \mathrm{HOAc}-\mathrm{H}_{2} \mathrm{O}=20 \%$ $(\mathrm{v} / \mathrm{v}), \mathrm{Temp}=50^{\circ} \mathrm{C}$

Table 1. Dependence of rate on the concentration of reactants

\begin{tabular}{|c|c|c|c|c|c|c|}
\hline \multirow[b]{2}{*}{ S.No. } & \multirow{2}{*}{$\begin{array}{c}{[\text { NBSA] }} \\
10^{3} \mathrm{M}\end{array}$} & \multirow{2}{*}{$\begin{array}{c}\text { [Acetophenone] } \\
10^{2} \mathrm{M}\end{array}$} & \multirow{2}{*}{$\begin{array}{c}{\left[\mathrm{HClO}_{4}\right]} \\
10^{2} \mathrm{M}\end{array}$} & \multicolumn{3}{|c|}{$\leftarrow 10^{3} \mathrm{k}_{1} \min ^{-1} \rightarrow$} \\
\hline & & & & $\begin{array}{l}\text { Without } \\
\text { micelle }\end{array}$ & $\begin{array}{c}\text { With } \\
\text { CTAB }\end{array}$ & $\begin{array}{l}\text { With } \\
\text { CTAB }\end{array}$ \\
\hline 1. & 0.83 & 2.0 & - & 14.59 & 21.60 & 25.91 \\
\hline 2. & 1.00 & 2.0 & - & 12.55 & 17.56 & 20.80 \\
\hline 3. & 1.25 & 2.0 & - & 8.50 & 14.50 & 19.16 \\
\hline 4. & 2.00 & 2.0 & - & 7.52 & 10.64 & 15.58 \\
\hline 5. & 2.50 & 2.0 & - & 4.88 & 6.60 & 8.75 \\
\hline 6. & 2.0 & 1.00 & - & 4.03 & 5.79 & 7.33 \\
\hline 7. & 2.0 & 1.25 & - & 4.83 & 6.68 & 9.39 \\
\hline 8. & 2.0 & 1.66 & - & 5.95 & 8.41 & 12.76 \\
\hline 9. & 2.0 & 2.50 & - & 8.01 & 11.52 & 17.56 \\
\hline 10. & 2.0 & 5.00 & & 11.19 & 15.73 & 31.36 \\
\hline 11. & 2.0 & 2.0 & 1.00 & 11.62 & 14.39 & 17.59 \\
\hline 12. & 2.0 & 2.0 & 2.00 & 13.61 & 16.34 & 18.45 \\
\hline 13. & 2.0 & 2.0 & 5.00 & 19.10 & 21.48 & 24.27 \\
\hline 14. & 2.0 & 2.0 & 7.50 & 24.44 & 26.79 & 28.34 \\
\hline 15. & 2.0 & 2.0 & 10.00 & 28.52 & 31.68 & 33.00 \\
\hline
\end{tabular}

$\left[\mathrm{Hg}(\mathrm{OAc})_{2}\right]=1.0 \times 10^{-2} \mathrm{M}, \mathrm{HOAc}-\mathrm{H}_{2} \mathrm{O}=20 \%(\mathrm{v} / \mathrm{v}),[\mathrm{NaLS}]=6.0 \times 10^{-6} \mathrm{M},[\mathrm{CTAB}]=5.0 \times 10^{-6} \mathrm{M}, \mathrm{Temp}=50^{\circ} \mathrm{C}$ 


\section{Dependence of rate on perchloric acid concentration}

For both micellar catalysed and uncatalysed reaction, the pseudo first order constant increases with increase in the concentration of perchloric acid (Table 1) suggesting that the reaction is acid catalysed. The slope value of log-log plot between $N$-bromosaccharin and $\left[\mathrm{HClO}_{4}\right]$ is $0.39,0.27$ and 0.40 for acetophenone in absence and presence of CTAB and $\mathrm{NaLS}$. The plot of $\mathrm{k}_{1}$ and $\left[\mathrm{HClO}_{4}\right]$ is linear with positive intercept suggesting that the reaction follows two paths, acid dependent and independent.

\section{Dependence of rate on solvent composition}

The effect of variation of dielectric constant of the medium was investigated by using acetic acid-water binary mixture of different compositions. The value of pseudo first order rate constant decreases with increase in the composition of acetic acid in the binary mixture for both oxidations i.e in the presence and absence of surfactant. The plot of $\log \mathrm{k}_{1}$ and inverse of dielectric constant of the medium is linear suggesting an interaction between dipole and positive ion or between two dipoles.

\section{Dependence of rate on saccharin concentration}

The reaction rate also decreases with increasing concentration of saccharin, one of the product. The plot of inverse of $k_{1}$ against [saccharin] is linear suggesting that saccharin is involved in some pre-equilibrium step.

The effect of addition of neutral salt on reaction rate was investigated by adding varying concentrations of potassium chloride. The increase in rate with increasing concentration of potassium chloride suggests that the reaction is positive ion- dipole. Under the concentration used for the present investigation mercuric acetate acts as scavenger for bromide ion. The reaction was studied at different temperatures and actvation parametrers were evaluated (Table 2).

\section{Reactive species and mechanism}

Like other haloamides and imides $\mathrm{N}$-bromosaccharin exists in various active forms such as the molecular species NBSA and the hydrolytic form $\mathrm{HOBr}$, their protonated forms $\mathrm{NBSAH}^{+}$and $\mathrm{HOBr}^{+}$and also as their acetate species.

$$
\begin{aligned}
\mathrm{NBSA}+\mathrm{H}^{\oplus} \longrightarrow \mathrm{NBSAH}^{\oplus} \\
\mathrm{NBSA}+\mathrm{H}^{\oplus} \longrightarrow \mathrm{Sac}+\mathrm{Br}^{\oplus} \\
\mathrm{NBSA}+\mathrm{H}_{2} \mathrm{O} \longrightarrow \mathrm{Sac}+\mathrm{HOBr}^{\oplus} \\
\mathrm{HOBr}+\mathrm{H}^{\oplus} \longrightarrow \mathrm{H}_{2} \mathrm{OBr}
\end{aligned}
$$

The effect of hydrogen ion concentration and effect of addition of varying concentration of saccharin on the oxidation rate play a key role in deciding the active oxidant and substrate species. In the present investigation the retardation of reaction rate with added saccharin suggests that saccharin is one of the products of pre-equilibrium hydrolysis of NBSA. It also rules out the involvement of molecular NBSA and protonated NBSA as active oxidant species.

$$
\mathrm{NBSA}+\mathrm{H}_{2} \mathrm{O} \longrightarrow \mathrm{Sac}+\mathrm{HOBr}
$$

The linearity of plot of inverse of $\mathrm{k}_{1}$ versus [saccharin] supports $\mathrm{HOBr}$ as active oxidant. The reactions are acid catalysed suggesting involvement of some protonated species viz. $\mathrm{H}_{2} \mathrm{O}^{+} \mathrm{Br}$ or $\mathrm{Br}^{+}$. 


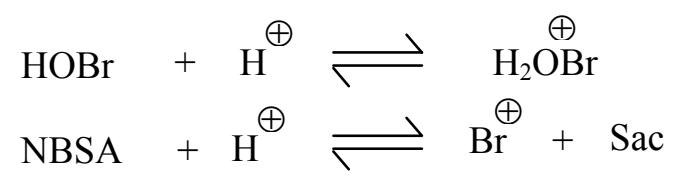

The above equation $(7 \& 8)$ explains both acid catalysis and inverse dependence of reaction rate on saccharin concentration but it is well established that $\mathrm{H}_{2} \mathrm{O}^{+} \mathrm{Br}$ is more effective electrophile than $\mathrm{Br}^{+}$although it is very difficult to distinguish between the two in aqueous solution. The formation and participation of hypobromous acidium ion $\mathrm{H}_{2} \mathrm{O}^{+} \mathrm{Br}$ is well documented in several oxidation reactions involving haloamides and imides as oxidants.

The nature of plot of $\mathrm{k}_{1}$ versus [perchloric acid] suggests that the oxidation in presence and absence of micelles follows two paths, an acid dependent and acid independent. Accordingly unprotonated hydrolytic product $\mathrm{HOBr}$ and its protonated form $\mathrm{H}_{2} \mathrm{O}^{+} \mathrm{Br}$ may be considered as active oxidant species involved in the oxidation.

In the oxidation the substrate, ketone molecule may participate in the keto form as well as enol form. Further the enolisation may or may not be a rate determining step. In the present investigation the oxidation of acetophenone is strictly first order ruling out enolisation as rate controlling step and also a measurement of the rate of enolisation determined by bromination studies showed it to be more rapid than rate of oxidation. The solvent effect studies and effect of adding neutral salts suggests a dipole-dipole or ion-dipole interaction in slow step. It has been already suggested that the active oxidant species is $\mathrm{HOBr}$ and $\mathrm{H}_{2} \mathrm{O}^{+} \mathrm{Br}$ hence active substrate molecule must be a neutral molecule i.e. the enol form.

Enols are hydroxyl compounds and most of the oxidations of hydroxyl compounds proceed via esterification. Such a mode of oxidation may be conceived for ketones as well. However, enols are acids of $\mathrm{pK}_{\mathrm{a}}$ comparable to phenols, their esterification would be small hence formation of transient intermediate complex may be suggested. The nature of double reciprocal plot between $\mathrm{k}_{1}$ and [substrate] gives kinetic evidence for the formation of transient complex, the hypobromite, between the oxidant species and enol form of the substrate.

In the present investigation, the addition of olefinic monomer such as acrylonitrile does not initiate polymerization suggesting the absence of free radicals as reaction intermediates.

Based on the above kinetic results, stoichiometry and product analysis a probable mechanism for the oxidation of acetophenone by $\mathrm{N}$-bromosaccharin in the absence of micelles has been proposed as:

$$
\begin{aligned}
& \text { Keto } \rightleftharpoons \text { Enol } \\
& \mathrm{NBSA}+\mathrm{H}_{2} \mathrm{O} \stackrel{\mathrm{K}_{1}}{\rightleftharpoons} \mathrm{HOBr}+\mathrm{Sac} \\
& \mathrm{HOBr}+\mathrm{H}^{\oplus} \stackrel{\mathrm{K}_{2}}{\rightleftharpoons} \underset{\mathrm{H}_{2} \mathrm{OBr}}{\stackrel{\oplus}{\rightleftharpoons}} \\
& \text { Enol }+\mathrm{HOBr} \stackrel{\mathrm{K}_{3}}{\rightleftharpoons} \underset{\text { Transient intermediate }}{\stackrel{\mathrm{X}_{1}}{\rightleftharpoons}+\mathrm{H}_{2} \mathrm{O}} \\
& \mathrm{X}_{1} \underset{\text { slow }}{\stackrel{\mathrm{k}_{4}}{\longrightarrow}} \text { Products }
\end{aligned}
$$

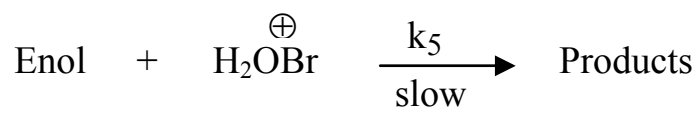




$$
\begin{gathered}
\text { Rate }=\mathrm{k}_{4}\left[\mathrm{X}_{1}\right]+\mathrm{k}_{5}[\mathrm{Enol}]\left[\mathrm{H}_{2} \mathrm{O}^{+} \mathrm{Br}\right] \\
\frac{\text { Rate }}{[\mathrm{NBSA}]_{\mathrm{T}}}=\frac{[\text { Enol }]\left\{\mathrm{K}_{1} \mathrm{~K}_{3} \mathrm{k}_{4}+\mathrm{K}_{1} \mathrm{~K}_{2} \mathrm{k}_{5}\left[\mathrm{H}^{+}\right]\right\}}{[\mathrm{Sac}]+\mathrm{K}_{1}+\mathrm{K}_{1} \mathrm{~K}_{2}\left[\mathrm{H}^{+}\right]+\mathrm{K}_{1} \mathrm{~K}_{3}[\text { Enol }]}
\end{gathered}
$$

\section{Oxidation of acetophenone in presence of micelles}

Micellar systems catalyse or retard the rate of chemical reactions. To investigate the effect of addition of surfactant to the acetophenone-NBSA system varying concentration of CTAB and NaLS were added. The plot of $\mathrm{k}_{1}$ versus [surfactant] depicts a curve with a maximum suggesting that the oxidation of acetophenone is micellar catalysed and biphasic i.e. oxidation proceeds in both aqueous and micellar phase. The kinetic results are explained by Piszkiewicz model. The plot of $\log \left[\left(\mathrm{k} \psi-\mathrm{k}_{\mathrm{W}}\right) /\left(\mathrm{k}_{\mathrm{M}}-\mathrm{k} \psi\right)\right]$ versus $\log [\mathrm{D}]$ is linear and the slope value "n", co-operative index, is calculated.

The rate of oxidation in presence of surfactant is of the order NaLS $>$ CTAB. This is also evident from the value of thermodynamic parameters tabulated in Table 2.

Table 2. Thermodynamic parameters

\begin{tabular}{lccccc}
\hline & $\begin{array}{c}\mathrm{E}_{\mathrm{a}} \\
\mathrm{JJ} \mathrm{mol}^{-1}\end{array}$ & $\begin{array}{c}\mathrm{A} \\
\mathrm{s}^{-1}\end{array}$ & $\begin{array}{c}\Delta \mathrm{H} \\
\mathrm{kJ} \mathrm{mol}^{-1}\end{array}$ & $\begin{array}{c}\Delta \mathrm{G}^{*} \\
\mathrm{~kJ} \mathrm{~mol}^{-1}\end{array}$ & $\begin{array}{c}\Delta \mathrm{S}^{*} \\
\mathrm{JK}^{-1} \mathrm{~mol}^{-1}\end{array}$ \\
\hline $\begin{array}{l}\text { Without } \\
\text { micelles }\end{array}$ & 71.98 & $3.19 \times 10^{7}$ & 67.05 & 93.38 & 79.69 \\
With CTAB & 60.31 & $6.678 \times 10^{5}$ & 57.11 & 92.64 & 107.49 \\
With NaLS & 49.27 & $1.542 \times 10^{4}$ & 46.09 & 92.07 & 139.13 \\
\hline
\end{tabular}

This may be explained by assuming that the attack of electrophilic oxidant species $\mathrm{H}_{2} \mathrm{O}^{+} \mathrm{Br}$ on enol is fasicilated to a some extent in the case of NaLS as compares to CTAB due to electrostatic attraction anionic micelles of NaLS can preferentially hold cationic oxidant species. Near constancy in the value of $\Delta \mathrm{G}^{*}$ for acetophenone-NBSA -surfactant and acetophenone-NBSA system suggest that they undergo by same mechanism. Addition of micelles of both cationic and anionic surfactants leads to a decrease in entropy and enthalpy of activation. This suggests greater stability of transition state in presence of surfactant as compared to its absence and the stabilization is even more in NaLS as compared to CTAB.

\section{Conclusion}

The kinetic oxidation studies of acetophenone by $\mathrm{N}$-bromosaccharin in presence of both cationic and anionic surfactants reveal that the oxidation is catalysed in presence of both the surfactants, NaLS is more effective than CTAB. The oxidations are acid catalysed and follow both acid dependent and independent path suggesting involvement of $\mathrm{HOBr}$ and $\mathrm{H}_{2} \mathrm{O}^{+} \mathrm{Br}$ as active oxidant species. Kinetic evidence also supports for the formation of intermediate complex between active oxidant and substrate species.

\section{References}

1. Kravchenko A I, Chem Abst., 1958, 52, 127544.

2. Tandon S K, Benerji K K and Bakore G K, Curr Sci., 1971, 40, 84.

3. Khandual N C, Nayak P L and Satpathy K K, (a) Indian J Chem., 1973, 8, 77; (b) J Chem Soc., 1974, 11, 328. 
4. Rao P L V S and Rao B S, J Indian Chem Soc., 1988, 65, 624.

5. Ganapathi K and Ramanujam M, Indian J Chem., 1980, 1319, 901.

6. Nath P and Benerji K K, Cand J Chem., 1970, 48, 2414.

7. Subramanian P V, Paul R and Subramanian V, Indian J Chem., 1973, 11, 1075.

8. Kelikar M S and Sankar R, Curr Sci., 1974, 43(24), 781.

9. Singh V P and Saxena S B, Indian J Chem., 1971, 9, 432.

11. Bacchawat J M and Mathur N K, Indian J Chem., 1971, 9, 1335-1336.

12. Barkat M Z and Abd-el-Wahab M F, Anal Chem., 1954, 26, 55.

13. Manoharan V and Venkatasubramanian N, Indian J Chem., 1984, 23, 389-391.

14. Sharma V K, Sharma K and Singh A, Oxid Commun., 1990, 13(4), 251.

15. Vijaymohan K, Manikyamba P, Raghunath Rao P and Sundram E V, Proc Natl Acad Sci., India, 1988, 58(A), 57.

16. Mishra U, Sharma K and Sharma V K, J Indian Chem Soc., 1986, 63, 586.

17. Sharma V K, Sharma K and Mishra U, Oxid Commun., 1988, 11(3-4), 275.

18. Shukla U, Sharma K and Sharma V K, Oxid Commun., 1992, 15, 219. 